\title{
MAGIC CIRCLES
}

BY C. G. P. KUSCHKE

If at every one of the $n^{2}+1$ points defined by $n$ concentric circles and $n$ radii is placed one of the first $n^{2}+1$ integers such that every circle with its center carries the same sum as every radius, I shall call this figure a system of magic circles of order $n$. Every magic square of order $n$ gives rise to two such circular systems, for the square with its $n$ rows and $n$ columns may be transformed into concentric circles, the rows becoming the circles and the columns the radii; all that is necessary is to assign to the center $C$ the number $n^{2}+1$ or 1 ; in the latter case all integers of the magic square must be raised by unity. Such systems I shall exclude here and all other systems I shall then call true circular systems.

The circles will be denoted by letters $a, b, c, \cdots$, ( $a$ being the smallest circle), and the radii by the integers $1,2,3, \cdots$ so that, for instance, the integer standing at the intersection of circle $d$ with radius 6 will be denoted by $d_{6}$.

All systems obtained by an interchange of rows, columns, or row and columns in any particular system will form a group and that system in the groups with $a_{1}=1, a_{2}<a_{3}<a_{4}<\cdots, b_{1}<c_{1}$ $<d_{1}<\cdots$, and $b_{1}<a_{2}$ will be considered the representative of this group. Every group contains, then, $2(n !)^{2}(1 / n)$ distinct systems and just one representative, because every system can belong to only one group and two systems are considered to be identical, if one may be obtained from the other by rotating merely the paper on which the figure is drawn. The sum of all integers in a system of order $n$ is

$$
S_{n}=\sum_{i=1}^{n^{2}+1} i=\frac{1}{2}\left(n^{2}+1\right)\left(n^{2}+2\right),
$$

and the magic sum is

$$
s_{n}=(1 / n)\left[S_{n}+(n-1) M\right]=1+b_{1}+c_{1}+d_{1}+\cdots,
$$

where $M$ is the element at the center, so that we have now two restrictions on $M$, namely, $1<M \leqq n^{2}$, and $M \equiv 1(\bmod n)$. For each $M$, all possibilities for the set $\left(b_{1}, c_{1}, d_{1}, \cdots\right)$ must be 
found and from the remaining integers in each case all chances for the set $\left(a_{2}, a_{3}, a_{4}, \cdots\right)$. What is left to be filled out may be stretched into a square of $n-1$ rows and $n-1$ columns, but that square would not be a magic square in the common sense for it has no magic constant; the magic number is a variable in the sense that, while every row and column must show a definite sum, this sum is different for any two rows and any two columns. I shall call such a square a variable magic square. Every such variable magic square of order $n-1$ gives rise to just one circular system of order $n$, for it must fit in this circular system so that no permutation of rows and columns is possible.

For $n=2$, there is no circular system, for our above conditions would lead to $b_{1}=a_{2}$. For $n=3$, we have $M \equiv 1(\bmod 3)$; therefore $M=4$ or 7 . If $M=4, s=21, b_{1}+c_{1}=16$, so that the pair $\left(b_{1}, c_{1}\right)=(6,10)$ or $(7,9)$. The pair $\left(b_{1}, c_{1}\right)=(6,10)$ gives $\left(a_{2}, a_{3}\right)$ $=(7,9)$, as $a_{2}+a_{3}=16$ too; and $\left(b_{1}, c_{1}\right)=(7,9)$ leaves no further room for $\left(a_{2}, a_{3}\right)$ under our above conditions for a representative. It remains, then, to fill out a variable magic square of order two; the rows must show the sums 11 and 7 , and the columns the sums 10 and 8 , and to do this we have for our disposal the integers $2,3,5,8$. It follows, then, that there is only one chance, namely, $b_{2}=8, b_{3}=3, c_{2}=2, c_{3}=5$. If $M=7, b_{1}+c_{1}=15$, so that $\left(b_{1}, c_{1}\right)=(5,10)$ and $\left(a_{2}, a_{3}\right)=(6,9)$. We have left for the variable magic square of order 2 the integers $2,8,3,4$, leading to only one chance, namely, $b_{2}=8, b_{3}=3, c_{2}=2, c_{3}=4$.

For $n=4, M=5,9$, or 13 . If $M=5, s_{4}=42$. There are 17 possibilities for the triad $\left(b_{1}, c_{1}, d_{1}\right)$, four of which leave no room for the triad $\left(a_{2}, a_{3}, a_{4}\right)$. The number of groups is 125 , so that we have $2(4 !)^{2}(1 / 4) 125=36000$ systems. For $M=9, s_{4}=45$. There are 18 possibilities for $\left(b_{1}, c_{1}, d_{1}\right)$, four of which give no triad $\left(a_{2}, a_{3}, a_{4}\right)$. The number of groups in this case is 517 . There are $2(4 !)^{2}(1 / 4) 517=148896$ circular systems corresponding to $M=9$. For $M=13, s_{4}=48$ and we have 17 possibilities for $\left(b_{1}, c_{1}, d_{1}\right)$, four of which admit no triad $\left(a_{2}, a_{3}, a_{4}\right)$. Here we have 117 groups and $2(4 !)^{2}(1 / 4) 117=33696$ circular systems.

Result. There are 48 true systems of magic circles of order 3 , 218592 of order 4 , and none of order 2.

University of Porto Rico 